\title{
Systems Neuroplasticity in the Aging Brain: Recruiting Additional Neural Resources for Successful Motor Performance in Elderly Persons
}

\author{
Sofie Heuninckx, Nicole Wenderoth, and Stephan P. Swinnen \\ Motor Control Laboratory, Group Biomedical Sciences, K.U. Leuven, B-3001 Heverlee, Belgium
}

\begin{abstract}
Functional imaging studies have shown that seniors exhibit more elaborate brain activation than younger controls while performing motor tasks. Here, we investigated whether this age-related overactivation reflects compensation or dedifferentiation mechanisms. "Compensation" refers to additional activation that counteracts age-related decline of brain function and supports successful performance, whereas "dedifferentiation" reflects age-related difficulties in recruiting specialized neural mechanisms and is not relevant to task performance. To test these predictions, performance on a complex interlimb coordination task was correlated with brain activation. Findings revealed that coordination resulted in activation of classical motor coordination regions, but also higher-level sensorimotor regions, and frontal regions in the elderly. Interestingly, a positive correlation between activation level in these latter regions and motor performance was observed in the elderly. This performance enhancing additional recruitment is consistent with the compensation hypothesis and characterizes neuroplasticity at the systems level in the aging brain.
\end{abstract}

Key words: aging; fMRI; motor control; interlimb coordination; cognition; compensation; dedifferentiation; neuroplasticity

\section{Introduction}

One of the most prominent challenges of current society is to develop a better understanding of the aging process. As a result of the demographic evolution in which elderly people occupy a gradually increasing cohort of the general population, it is of high socioeconomic importance to promote functional independence and comfort of living in this group. This requires a profound knowledge of the processes of neural aging. Recently, imaging studies have shown that seniors exhibit stronger brain activation than younger controls during the execution of various motor tasks. Old subjects activate the same regions as their younger counterparts, but to a larger extent, and/or they activate additional regions that are not observed in the young subjects ( $\mathrm{Ca}-$ lautti et al., 2001; Mattay et al., 2002; Ward and Frackowiak, 2003; Heuninckx et al., 2005). Although this "overactivation" in the aging brain is well documented within the motor system, the underlying neural mechanisms are still unclear. Based on previous results in cognitive aging studies, we put forward two major hypotheses. On the one hand, the compensation hypothesis predicts that age-related increases in brain activation, as well as the recruitment of additional areas, compensate for various neural/ behavioral deficits (e.g., neurodegeneration, attentional prob-

Received Feb. 15, 2007; revised 0ct. 4, 2007; accepted Nov. 9, 2007.

This work was supported by a grant from the Research Council of K.U. Leuven, Belgium (Contract 0T/07/73) and the Research Program of the Research Foundation-Flanders (FW0) (G.0460.04 and G.0105.00). S.H. was supported by a PhD fellowship from FW0.

Correspondence should be addressed to Dr. Stephan P. Swinnen, Laboratory of Motor Control, Division of Motor Control and Neuroplasticity, Department of Biomedical Kinesiology, Group Biomedical Sciences, K.U. Leuven, Tervuursevest 101, B-3001 Heverlee, Belgium. E-mail: Stephan.Swinnen@faber.kuleuven.be.

DOI:10.1523/JNEUROSCI.3300-07.2008

Copyright $\odot 2008$ Society for Neuroscience $\quad$ 0270-6474/08/280091-09\$15.00/0 lems, reduction in sensory function, etc.) (Madden et al., 1999; Reuter-Lorenz et al., 2000; Cabeza, 2002; Cabeza et al., 2002; Grady, 2002; Reuter-Lorenz and Lustig, 2005). On the other hand, the dedifferentiation hypothesis assumes that age-related changes in functional activation reflect a generalized nonfunctional spread of activity attributable to deficits in neurotransmission, which in turn causes a decrease in the signal-to-noise ratio in neural firing and a loss of neural specialization ( $\mathrm{Li}$ and Lindenberger, 1999).

Here, we aimed to unravel whether the overactivation in the motor networks of the elderly results from compensation or dedifferentiation on brain level. Functional magnetic imaging was applied to 24 older adults and 11 young controls to register brain activity during the performance of rhythmical hand-foot coordination tasks, whereby both limbs moved either in the same (isodirectional) or in the opposite (nonisodirectional) direction. Previous behavioral work showed convincingly that the nonisodirectional pattern is more difficult and is produced with lower accuracy and stability than the isodirectional pattern (Baldissera et al., 1982, 1991; Kelso and Jeka, 1992; Carson et al., 1995; Swinnen et al., 1995; Serrien et al., 2000).

Subsequently, activation in dedicated brain regions was correlated with motor performance in the elderly. According to the compensation hypothesis, the underlying rationale was that the overactivation would be larger in good than in poor motor performers, with the effect being more pronounced in more (nonisodirectional) than less (isodirectional) demanding coordination tasks. Conversely, the dedifferentiation hypothesis assumed overactivation to be larger in poor than in successful motor performers because of nonfunctional neural irradiation. Thus, positive correlations between brain activation and motor perfor- 


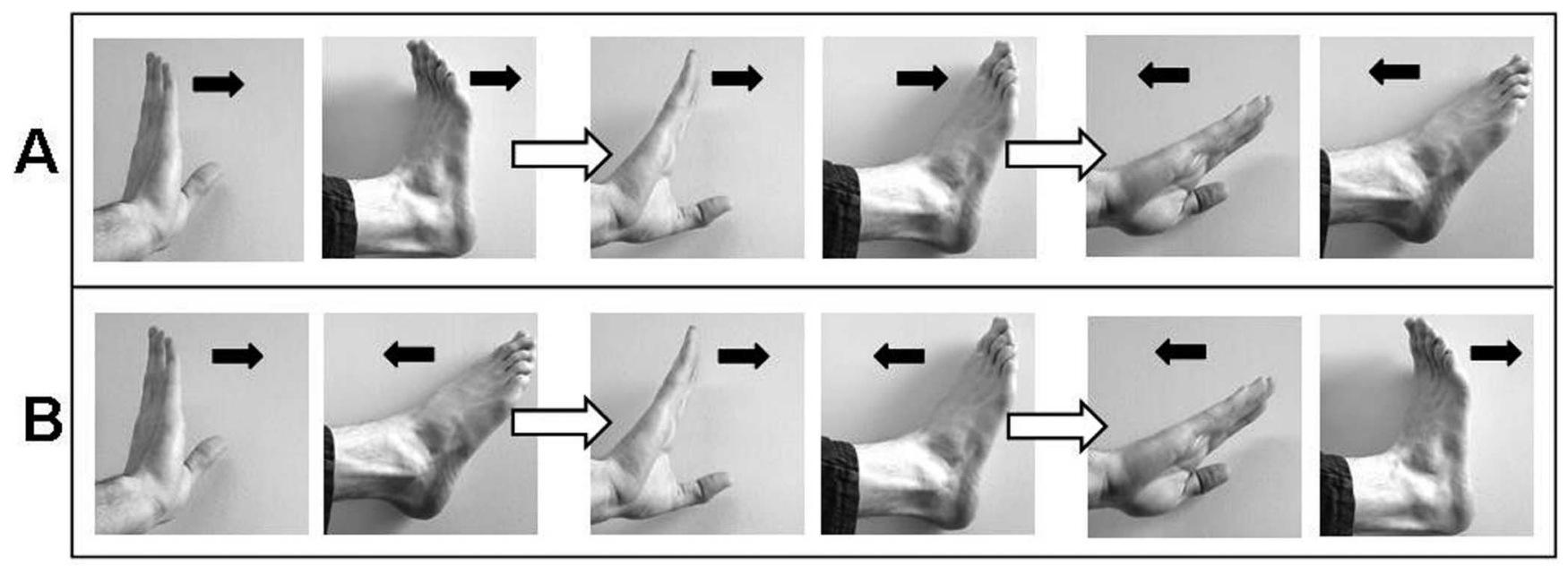

Figure 1. Cyclical ipsilateral coordination of the hand and foot according to the isodirectional mode ( $\boldsymbol{A}$; both limb segments are moved in the same direction) and the nonisodirectional mode ( $\boldsymbol{B}$; both limb segments are moved in opposite directions).

mance were considered to reflect compensation, and negative correlations were considered to reflect dedifferentiation.

\section{Materials and Methods \\ Participants}

Twelve young adults (mean age, 22.4 years; range, 20-25 years; 6 women and 6 men) and 26 older adults (mean age, 65.7 years; range, 62-72 years; 12 women and 14 men) participated in the study. The older subjects were all community-dwelling individuals. All participants were right-handed, as assessed by the Edinburgh Handedness Inventory (Oldfield, 1971). None reported a history of neurological disease or were taking psychoactive or vasoactive medication. General cognitive functions were assessed using the Mini-Mental State Examination (Folstein et al., 1975). All participants scored within normal limits (score 226 ). Participants were informed about the experimental procedures and provided written informed consent. The study was approved by the local ethics Committee of Biomedical Research at Katholieke Universiteit Leuven and was performed in accordance with the ethical standards of the 1964 Declaration of Helsinki. Imaging data of one young and two older subjects contained artifacts and were excluded from all analyses.

\section{Experimental design}

Task

During scanning, the participants performed three different conditions: two movement conditions requiring cyclical coordination of the right hand and right foot according to either the isodirectional (ISODIR) or the nonisodirectional (NONISODIR) mode, and one rest (REST) condition in which no movements were performed. During ISODIR coordination, both limb segments were moved in the same direction (i.e., hand flexion together with foot flexion) (Fig. $1 A)$. During NONISODIR coordination, segments were moved in opposite directions (i.e., hand flexion combined with foot extension) (Fig. 1B). Additionally, the participants also performed cyclical flexion-extension movements of the right wrist and right ankle separately. However, the latter movement conditions will not be further addressed here.

During scanning, participants lay supine in the scanner. The lower legs were supported by a cushion to ensure free ankle rotation. The right arm was extended along the trunk, and the distal part of the arm was supported to enable free movements of the wrist. A bite-bar was used to minimize head motion. In this position, subjects looked at a display via a mirror (at a distance of $36 \mathrm{~cm}$ from their eyes) onto which a visual template, displaying the task to be performed, was provided by means of a Barco (Kortrijk, Belgium) 6400i liquid crystal display projector $(1024 \times 768$ pixels, $60 \mathrm{~Hz})$. The wrist and foot were positioned in a nonferromagnetic wrist-hand and ankle-foot orthosis, respectively. Movements were restricted to the sagittal plane. The frictionless axis of the orthosis was aligned with the anatomical axis of the joint such that movements were not hindered. Angular displacements of the joints were registered by means of high-precision shaft encoders ( 4096 pulses per revolution; sampled at $100 \mathrm{~Hz}$ ) fixed to the movement axis of the orthosis. The nonferromagnetic kinematic registration device enabled us to register movements on-line during brain scanning. Movements were limited to the wrist and ankle, whereas the other segments were kept still. Subjects were trained to look at a fixation cross, displayed in front of them at all times.

All conditions were paced by an electronic metronome (DTM-12; KORG, Tokyo, Japan), whereby a full movement cycle was completed on every beat (one beat for peak flexion and one for peak extension). The older adults performed five scanning runs at a movement frequency of 1 $\mathrm{Hz}$, whereas the younger adults performed 10 runs, five at a movement frequency of $1 \mathrm{~Hz}$ and five at a movement frequency of $1.5 \mathrm{~Hz}$. For purposes of equating difficulty level, however, we compared the older subjects' performance at $1 \mathrm{~Hz}$ to that of the younger subjects' performance at $1.5 \mathrm{~Hz}$. Indeed, previous work demonstrated that these cycling frequencies represented a comparable ratio to the maximal frequency at which these patterns could be performed successfully by both groups (Heuninckx et al., 2004). Between the different scanning runs, rest periods of 3 min were provided.

Before scanning took place, a 45 min practice session was provided in a dummy scanner to ensure correct performance. Subjects were trained to avoid eye movements and to look at a fixation cross.

\section{Scanning procedure}

The magnetic resonance (MR) images were acquired in a $3 \mathrm{~T}$ Intera MR scanner (Philips, Best, The Netherlands), using a six-element SENSE head coil (MRI Devices, Waukesha, WI). Functional time series consisted of 105 whole-brain gradient-echo echoplanar images (EPIs) [repetition time (TR), $3000 \mathrm{~ms}$; echo time (TE), $33 \mathrm{~ms}$; field of view, $230 \mathrm{~mm}$; matrix, $112 \times 112$; slice thickness, $4.0 \mathrm{~mm}$; interslice gap, $0.4 \mathrm{~mm} ; 34$ sagittal slices; SENSE factor, 2]. Each time series contained three blocks of the five conditions. Each condition lasted $21 \mathrm{~s}$ (corresponding to seven whole-brain images) and was triggered by a visual template displaying the task to be performed. The different task conditions were randomized across subjects and runs. Each scanning session ended with the acquisition of a three-dimensional SENSE high-resolution, T1-weighted image (TR, $9.68 \mathrm{~ms}$; TE, $4.6 \mathrm{~ms}$; inversion time, $1100 \mathrm{~ms}$; field of view, $250 \mathrm{~mm}$; matrix, $256 \times 256$; slice thickness, $1.2 \mathrm{~mm}$; 182 slices; SENSE factor, 2) for anatomical detail. 
Table 1. Kinematic results

\begin{tabular}{clllll}
\hline & \multicolumn{2}{l}{ Old } & & \multicolumn{1}{l}{ Young } & NONISODIR \\
\cline { 2 - 3 } \cline { 5 - 6 } & ISODIR & NONISODIR & & ISODIR & \\
\hline AE mean & $22.85^{\circ}$ & $26.18^{\circ}$ & & $18.7^{\circ}$ & $22.9^{\circ}$ \\
Range & $\left(14.94-32.28^{\circ}\right)$ & $\left(16.71-46.40^{\circ}\right)$ & & $\left(15.88-22.96^{\circ}\right)$ & $\left(18.2-28.34^{\circ}\right)$ \\
SD mean & $18.5^{\circ}$ & $22.9^{\circ}$ & $14.5^{\circ}$ & $18.4^{\circ}$ \\
Range & $\left(15.19-29.83^{\circ}\right)$ & $\left(16.09-42.14^{\circ}\right)$ & & $\left(13.21-15.58^{\circ}\right)$ & $\left(15.76-21.47^{\circ}\right)$ \\
\hline
\end{tabular}

\section{Data analyses}

\section{Kinematic analyses}

The coordination between the limb segments was assessed by means of a relative phase measure, that is the subtraction of the phase angles of each limb according to the following formula: $\Phi=\theta_{\mathrm{w}}-\theta_{\mathrm{f}}=\tan ^{-1}\left[\left(d X_{\mathrm{w}} /\right.\right.$ $\left.d t) / X_{\mathrm{w}}\right]-\tan ^{-1}\left[\left(d X_{\mathrm{f}} / d t\right) / X_{\mathrm{f}}\right]$, where $w$ and $f$ are wrist and foot, respectively; $\theta_{\mathrm{w}}$ is the phase of the wrist movement at each sample; $X_{\mathrm{w}}$ is the position of the wrist after rescaling to the interval $[-1,1]$ for each cycle of oscillation; and $d X_{\mathrm{w}} / d t$ is the normalized instantaneous velocity. Absolute deviations from the target relative phase (i.e., 0 and $180^{\circ}$ for ISODIR and NONISODIR coordination, respectively) were calculated to obtain a measure of relative phase accuracy ( $\mathrm{AE} \Phi$, phase error). The $\mathrm{SD}$ of relative phase (SD $\Phi)$ was used as an estimate of movement pattern stability.

For the statistical analysis, the aforementioned parameters were determined for each condition and subsequently averaged across repetitions and runs. The statistical analyses consisted of repeated-measures ANOVA with the between-factor group (young, old) and the withinfactor mode (ISODIR, NONISODIR). The $\alpha$ level of significance was set to $\alpha=0.05$.

\section{Imaging analysis}

Imaging data were analyzed with Statistical Parametric Mapping 2 (SPM2) (Wellcome Department of Imaging Neuroscience, London, UK) implemented in MatLab 6.5 (MathWorks, Natick, MA). For each subject, all EPI volumes were realigned to the first volume of the first time series, and a mean image of the realigned volumes was created. This mean image was smoothed with a Gaussian kernel of $6 \mathrm{~mm}$ full-width at halfmaximum (FWHM) and coregistered to the anatomical T1-weighted image. To normalize the anatomical image as well as the EPIs to a standard reference system (Talairach and Tournoux, 1988), the following procedure was applied. First, the anatomical image as well as a representative template image [Montreal Neurological Institute (MNI)] was segmented into gray matter, white matter, and CSFs. Then, the anatomical gray matter image was smoothed ( $6 \mathrm{~mm}$ FWHM) and normalized to the gray matter of the MNI brain. Subsequently, the derived normalization parameters were applied to the EPIs, which were subsampled to a voxel size of $2 \times 2 \times 2 \mathrm{~mm}$ and smoothed with a Gaussian kernel of $10 \mathrm{~mm}$ FWHM.

All statistical analyses were performed in the context of the general linear model (Friston et al., 1995a,b). Each condition was modeled using a delayed boxcar function convolved with the SPM2 hemodynamic response function. An appropriate high-pass filter was applied to remove low-frequency drifts. Additionally, movement parameters derived from realignment were added as covariates of no interest to correct for confounding effects induced by head movement. Contrasts of interest were first estimated for each subject individually (averaging activation across runs) and then subjected to a second-level random-effects analysis.

Between-group conjunction: regions similarly activated in the young and the elderly. We investigated which regions were similarly activated in both age groups during ISODIR and NONISODIR coordination, respectively. First, within-group activations were calculated by contrasting each movement condition with REST, and subsequently, the following

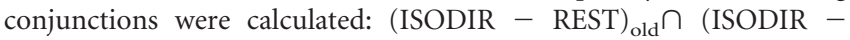
REST) $)_{\text {young }}$ and (NONISODIR - REST) ${ }_{\text {old }} \cap(\text { NONISODIR }- \text { REST) })_{\text {young }}$ for ISODIR and NONISODIR coordination, respectively. Conjunctions were determined in accordance with the method suggested by Nichols et al. (2005). A positive conjunction test implies that a given region is significantly activated by each age group. Additionally, our further analysis revealed that none of these commonly activated areas differed significantly between groups, indicating that the regions exhibited a similar activation level in the young and in the elderly. A false discovery rate (FDR) correction was applied, ensuring an overall $p<0.05$ (i.e., $t>$ 4.00). Only clusters with a size of $\geq 20$ voxels will be reported.

Between-group analysis: determining differentially activated regions. To determine which regions were differentially activated in old versus young subjects for ISODIR and NONISODIR coordination, between-group comparisons were made by means of independent $t$ tests. The following contrasts were calculated: (ISODIR - REST) $)_{\text {old }}$ versus (ISODIR - REST) young $_{\text {and }}$ $(\text { NONISODIR }- \text { REST) })_{\text {old }}$ versus $(\text { NONISODIR }- \text { REST) })_{\text {young }}$ FDR correction was applied, ensuring an overall $p<0.05$ (i.e., $t>4.00$ ). Only clusters with a size of $\geq 20$ voxels will be reported.

Within-group analyses: correlation between motor performance and brain activation with respect to similarly and differentially activated regions. Within both age groups, multiple regression analyses were performed to investigate which brain regions exhibited a significant correlation with performance on the coordination task across subjects. For the ISODIR - REST contrast as well as for the NONISODIR - REST contrast, separate second-level models were defined, containing the performance level for each individual as well as age as a covariate of no interest. Coordination performance was quantified by means of (1) the absolute phase error (coordination accuracy) and (2) the SD of relative phase (coordination stability). To simplify interpretation, the relationship between error scores and performance was inverted (1/AE and 1/SD, respectively) such that high scores were indicative of good performance. In a first step, we identified all regions exhibiting a significant positive or negative correlation with performance $(p<0.001 ; t>2.52$; uncorrected for multiple comparisons). Within this performance-dependent network, we applied small-volume corrections (FDR, $p<0.05, t>2.86$ ) within the clusters that were either (1) similarly activated by the young and elderly (i.e., clusters of the between-group conjunction reaching significance on cluster level with $p<0.05$ ) or (2) differentially activated in the young and elderly (i.e., clusters of the between-group analysis that were more strongly activated in the elderly than in the young, and vice versa, reaching significance on cluster level with $p<0.05$ ).

\section{Results}

\section{Kinematic data}

Separate group (young, elderly) $\times$ coordination mode (ISODIR, NONISODIR) ANOVAs with repeated measures on the last factor were conducted on relative phase error and SD of relative phase, respectively. Both analyses revealed significant main effects of coordination mode (AE: $F_{(1,33)}=16.5, p<0.0005$; SD: $\left.F_{(1,33)}=31.5, p<0.0001\right)$ and group $\left(\mathrm{AE}: F_{(1,33)}=5.7, p<0.05\right.$; SD: $\left.F_{(1,33)}=26.4, p<0.0001\right)$. In accordance with the literature, ISODIR coordination was performed with higher accuracy and stability than NONISODIR coordination (Table 1). Although different cycling frequencies were imposed to equate task difficulty level across both groups, coordination performance of older participants was slightly less accurate and stable than that of the younger subjects (Table 1).

The range of $\mathrm{AE}$ and $\mathrm{SD}$ scores in the younger subjects was fairly small with all subjects having comparable performance levels (Table 1). In the elderly group, however, the range was much larger, with some elderly performing poorly and others performing as well as the younger controls (Table 1).

\section{fMRI data}

Between-group conjunction: regions similarly activated in the young and elderly

Brain regions that were activated by both age groups to a similar extent were identified for the ISODIR and NONISODIR coordi- 
nation modes, respectively. During the ISODIR coordination mode, both groups activated a typical coordination network, including the contralateral precentral and postcentral gyri and the paracentral lobule, corresponding to the primary sensorimotor cortex (SM1), the left and right supplementary motor area (SMA), the left cingulate motor area (CMA), and the left and right lateral sulcus/posterior insula, corresponding to the secondary somatosensory area (S2). Significant subcortical activation was present in the contralateral thalamus, putamen, and pallidum and in the ipsilateral anterior cerebellum (for coordinates and $t$ values, see supplemental Table $1^{*}$, available at www.jneurosci.org as supplemental material).

For the NONISODIR coordination pattern, similar results were observed (i.e., brain regions similarly activated in both age groups were the left SM1, CMA, and SMA; the left and right S2; and subcortically, the left thalamus and pallidum and the right anterior cerebellum) (Fig. 2; for coordinates and $t$ values, see supplemental Table $1^{*}$, available at www.jneurosci.org as supplemental material).

Within-group analyses: correlation between motor performance and brain activation with respect to the similarly activated regions

In this section, areas are reported that exhibit a significant correlation with performance and are similarly activated in both age groups. Motor performance was quantified by means of the absolute phase error (coordination accuracy) and the SD of relative phase (coordination stability), respectively. However, because relative phase error and SD were highly correlated in the elderly (Pearson's $r=0.89$ and $r=0.86$ for ISODIR and NONISODIR, respectively) and appreciable in the younger adults (Pearson's $r=0.50$ and $r=$ 0.62 for ISODIR and NONISODIR, respectively), it is not surprising that very similar results were obtained for both measures. Therefore, we decided to only report the results using the phase error scores as regressor.

In the elderly, a significant positive correlation between brain activation and coordination performance on the relatively easy ISODIR coordination task was observed in the left precentral and postcentral gyri (SM1) ( $p<0.05$, cluster-wise FDR correction). Additionally, there was a trend ( $p<0.001$, uncorrected) toward a positive correlation in the right anterior cerebellar hemisphere (Table 2). This suggests that the more the ISODIR coordination task was performed successfully in elderly subjects, the higher the levels of brain activation. In none of the similarly activated regions was a negative relationship between brain activation and ISODIR performance obtained.

In the young subjects, no significant relationship between level of brain activation and performance on the ISODIR coordination task was observed.

In the elderly, a significant positive correlation between brain activation and coordination performance on the more demand- ing NONISODIR coordination task was observed in the left superior postcentral gyrus/sulcus (Fig. $2 A$ ) and inferior postcentral gyrus (Fig. $2 B$ ). Additionally, positive correlations were also obtained in the right SMA (Fig. $2 C)$ and in the left CMA $(p<0.05$, cluster-wise FDR correction) (Fig. $2 D$; see Table 2 for summary). In Figure 2, these areas are indicated by an arrow. The graphics display each subject's blood oxygenation level-dependent (BOLD) response for the within-cluster peak activation, as a function of the inverse of phase error (1/AE), with the younger subjects in blue and the older subjects in red. It can be inferred that, on average, the elderly and young subjects exhibited similar BOLD responses. However, the range of BOLD responses was smaller in the young than elderly group. More specifically, the well performing elderly showed equal or higher BOLD responses than the young controls, whereas the poor performing elderly exhibited lower BOLD responses than the young.

In the other brain regions, similarly activated in both groups (i.e., the left central sulcus/precentral gyrus, paracentral lobule, S2, thalamus, and pallidum and the right anterior cerebellum), no significant relationships between level of brain activation and performance on the NONISODIR coordination task were observed in the elderly group ( $p>0.001$, uncorrected). In none of the similarly activated regions were significant negative relationships between brain activation and NONISODIR performance obtained. 


\begin{tabular}{|c|c|c|c|c|c|}
\hline Region activated & Side & $x$ & $y$ & $z$ & $t$ value \\
\hline \multicolumn{6}{|l|}{ ISODIR coordination } \\
\hline Precentral gyrus (M1 hand area) & L & -44 & -12 & 60 & 3.90 \\
\hline Postcentral gyrus (S1 hand area) & L & -46 & -30 & 60 & 3.92 \\
\hline Cerebellar hemisphere (V) & $R$ & 24 & -54 & -18 & 2.84 \\
\hline \multicolumn{6}{|l|}{ NONISODIR coordination } \\
\hline SMA & $M / R$ & 8 & -16 & 58 & 3.79 \\
\hline Inferior postcentral gyrus & L & -38 & -26 & 46 & 3.74 \\
\hline Cingulate cortex (CMA) & Ml & -44 & -12 & 60 & 3.90 \\
\hline Postcentral gyrus/sulcus & $\mathrm{L}$ & -46 & -30 & 60 & 3.92 \\
\hline
\end{tabular}

The $t$ values and localizations (MNI coordinates) of activation peaks showing a significant ( $p<0.05$; cluster-wise FDR correction for multiple comparisons) positive correlation between level of brain activity and coordination performance are shown. Regions reaching significance only at an uncorrected level $(p<$ 0.001 ) are in italics. L, Left hemisphere; R, right hemisphere; M, medial; M1, primary motor cortex; $\mathrm{S1}$, primary sensory cortex.

In the young subjects, no significant relationship between level of brain activation and performance on the NONISODIR coordination task was observed.

\section{Between-group analysis: determining differentially activated regions}

Between-group comparisons were made by means of independent $t$ tests for the ISODIR and NONISODIR coordination mode, respectively. During the ISODIR coordination mode, the elderly group exhibited significantly higher activation than the young group in the left anterior insular cortex (for coordinates and $t$ values, see supplemental Table $2^{*}$, available at www. jneurosci.org as supplemental material).

No region was significantly more activated in the younger than older group.

During the NONISODIR coordination mode, the pattern of activation in the old group was more widespread than in the young group (Fig. 3; for coordinates and $t$ values, see supplemental Table $2^{*}$, available at www.jneurosci.org as supplemental material). The old group exhibited significantly higher activation in the left anterior insular cortex, inferior frontal gyrus pars opercularis (IFGPO), and inferior frontal gyrus pars triangularis (IFGPT). In addition, larger activation for the old group was observed in the left middle frontal gyrus, corresponding to the dorsolateral prefrontal cortex (DLPFC), and in the left superior frontal sulcus and gyrus, corresponding to the anterior dorsal premotor area (pre-PMd). Larger activation for the old group was also observed in the left superior temporal gyrus, angular gyrus, superior parietal gyrus, fusiform gyrus, and inferior postcentral sulcus, corresponding to S2, and in the left and right lingual gyrus. In the right hemisphere, the older group demonstrated significantly larger activation in the paracentral lobule and parahippocampal gyrus. Finally, the old group exhibited significantly higher activation than the young group in the left and right anterior cerebellum and in the right posterior cerebellum.

No region was significantly more activated in the younger than in the older group.

For a detailed description of differences in brain activation during ISODIR versus NONISODIR coordination, in young versus old subjects, see Heuninckx et al. (2005).

Within-group analyses: correlations between motor performance and brain activation with respect to differentially activated regions In this section, areas are reported that exhibit a significant correlation with performance and are significantly overactivated in the elderly.

For the ISODIR coordination mode, no significant relationships between activation and coordination accuracy were identi- fied. In contrast, for the more complex NONISODIR coordination mode, significant positive relationships were observed in the left IFGPO and IFGPT (Fig. 3B), anterior insular cortex (Fig. $3 C$ ), superior parietal gyrus (Fig. $3 F$ ), pre-PMd (Fig. $3 E$ ), and DLPFC (Fig. 3A) and in the right posterior (Fig. $3 H$ ) and left anterior (Fig. 3D) cerebellar hemisphere $(p<0.05$, cluster-wise FDR correction). Additionally, there was a trend ( $p<0.001$, uncorrected) toward a positive correlation in the left superior temporal gyrus (Fig. 3G; see Table 3 for summary). In Figure 3 , the brain regions that were additionally activated by the elderly subjects while showing a positive relationship between activation and coordination performance are indicated by an arrow. The individual BOLD responses with respect to the within-cluster peak activation in the old (red) and young (blue) subjects are displayed as a function of the inverse of phase error (1/AE). It can be observed that the elderly performing poorly exhibited a similar BOLD response as the young controls, whereas the more successful elderly clearly exhibited higher levels of brain activation.

In the other differentially activated regions (i.e., the left S2, fusiform gyrus, angular gyrus, lingual gyrus, posterior cerebellum, right parahippocampal gyrus, and paracentral lobule), no significant relationships were identified. In none of the differentially activated regions were negative relationships observed.

The scatter plots in Figures 2 and 3 show one older adult whose performance was much worse than the remaining ones during the NONISODIR coordination task. Additional analyses with exclusion of this subject revealed highly similar results, suggesting that the present correlations are robust (see supplemental Tables $3^{\star}, 4^{\star}$, available at www.jneurosci.org as supplemental material).

\section{Discussion}

The aim of the present study was to investigate whether agerelated overactivation during motor performance reflects compensation or dedifferentiation mechanisms. According to the compensation hypothesis, overactivation counteracts age-related decline of brain function and supports performance (Madden et al., 1999; Reuter-Lorenz et al., 2000; Cabeza, 2002; Cabeza et al., 2002; Grady, 2002; Reuter-Lorenz and Lustig, 2005). According to the dedifferentiation hypothesis, overactivation may be nonfunctional, reflecting age-related difficulties in recruiting specialized neural mechanisms ( $\mathrm{Li}$ and Lindenberger, 1999), and is therefore either irrelevant for the task or associated with bad performance. To contrast predictions from both hypotheses, performance on coordination tasks with different levels of complexity was correlated with brain activation in older subjects. This approach was applied to brain regions that were (1) similarly activated in both age groups or (2) more activated in the elderly compared with the young group (e.g., compensatory recruitment).

The kinematic data revealed that coordination accuracy varied substantially in the elderly, with some performing poorly and others performing as well as the younger controls.

Our main finding is that the elderly exhibited a significant correlation between activation in dedicated brain regions and performance, such that good performers exhibited higher brain activation levels than poor performers. This effect was more prevalent during 
the more demanding nonisodirectional than during the easier isodirectional coordination task. Accordingly, our results provided strong support for the compensation hypothesis. Interestingly, this positive association between performance and brain activity was not evident across the entire brain but rather in some specific, well defined regions that were either recruited by both age groups or additionally recruited by the elderly compared with the young group.

Regions similarly activated by both age groups and exhibiting a positive association between brain activity level and motor performance

In the elderly, significant positive correlations were observed between motor performance and activation in the contralateral SM1 and ipsilateral anterior cerebellum during the ISODIR coordination mode and in the contralateral SM1, SMA, and CMA during the more difficult NONISODIR coordination mode. These regions were activated by both age groups and represent typical motor regions that are usually activated by ipsilateral hand-foot movements (Ehrsson et al., 2000; Debaere et al., 2001; Heuninckx et al., 2005). Increased activations of SM1, SMA, CMA, and cerebellum have repeatedly been observed in multilimb coordination tasks (Debaere et al., 2004; for review, see Wenderoth et al., 2004b). Especially SMA and cerebellar activity are hypothesized to reflect increasing demands on motor timing (Ivry, 1997; Macar et al., 1999; Mima et al., 1999; Habas et al., 2004; Wenderoth et al., 2004b) and/or sensory processing (Jueptner et al., 1997; Bushara et al., 2001; Thickbroom et al., 2003; Debaere et al., 2004). Therefore, the observed positive correlations between coordination performance and brain activity in these motor regions may reflect a successful compensatory response to increased functional demands by the high-performing elderly.

Overactivated regions in the elderly, exhibiting a positive association between brain activity level and motor performance

Interestingly, besides typical motor regions, more remote regions were also additionally recruited in the elderly and correlated positively with performance on the coordination task.

First, positive correlations were observed in the contralateral superior parietal cortex, contralateral posterior cerebellum, and ipsilateral anterior cerebellum, which were previously shown to be involved in higher-order sensorimotor coordination during more demanding tasks (Debaere et al., 2004; Wenderoth et al., 2004b). Whereas the cerebellum is a typical timing and coordination organ (see above), the superior parietal cortex is strongly involved in sensorimotor integration and spatial aspects of movement planning (Wenderoth et al., 2004a,b, 2005, 2006). Both

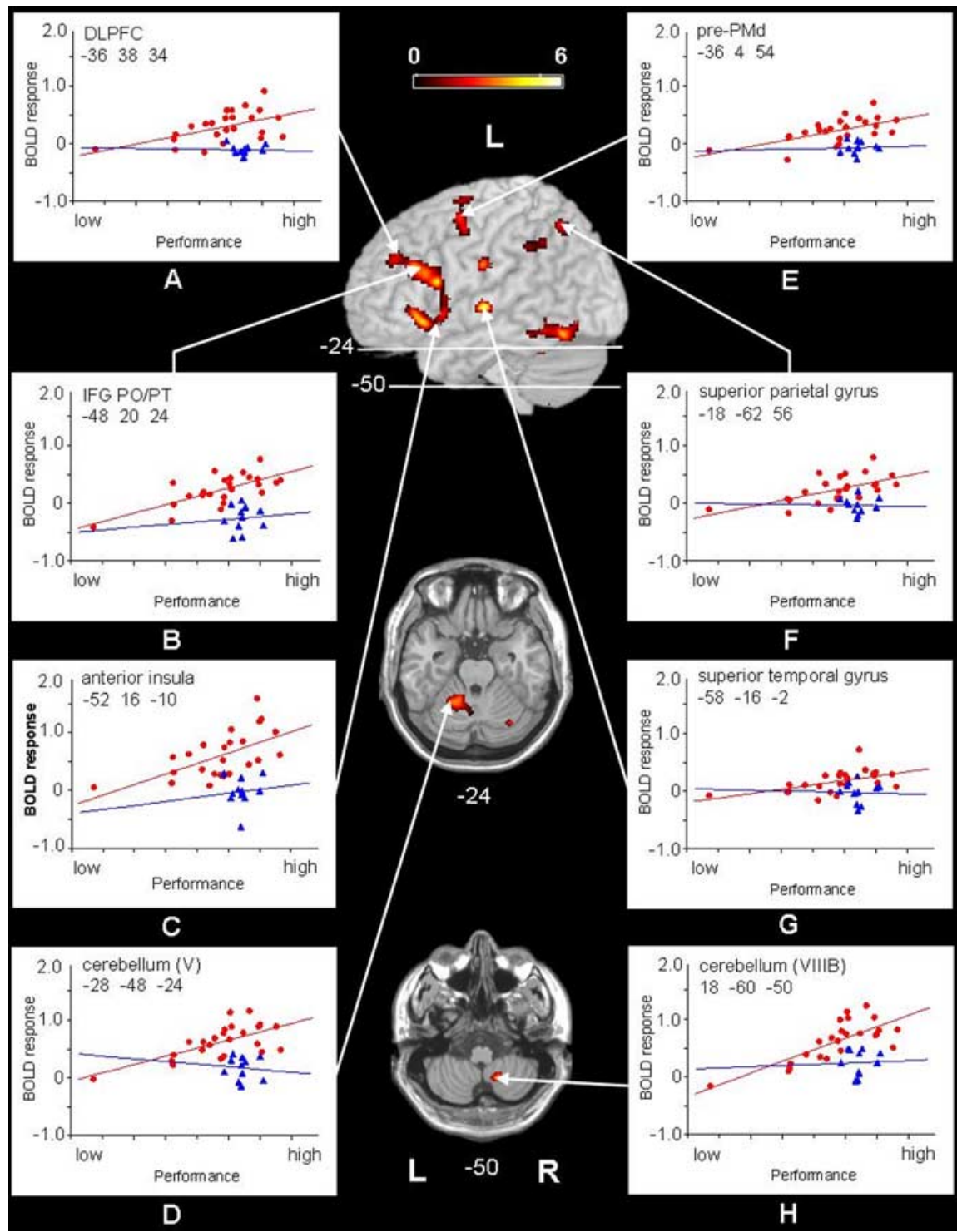

Figure 3. Statistical parametric maps representing significantly larger activation in the old compared with the young group

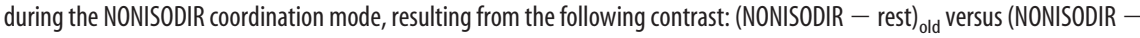

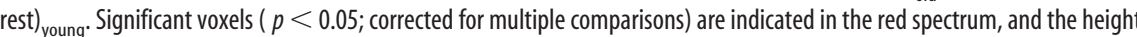
correlation between brain activity level and coordination performance, as identified by a whole-brain multiple regression analysis followed by a small-volume correction with the clusters, overactivated by the elderly as shown in the statistical parametric subject's BOLD response with respect to the within-cluster peak activation as a function of the inverse of the phase error (1/AE) with the younger subjects in blue and the older subjects in red.

spatial and temporal integration are critical features of successful interlimb coordination.

Second, we also identified several nonmotor regions that were additionally recruited by the elderly as well as related to successful motor performance. We identified relatively large clusters surrounding the left frontal operculum, with peak activation in the anterior insular cortex, IFGPO, IFGPT, and superior temporal gyrus. The IFGPO, anterior insula and superior temporal gyrus appear to be involved in higher-order auditory processing (Platel et al., 1997; Bamiou et al., 2003; Thaut, 2003) and particularly in motor synchronization to an auditory rhythm (Thaut, 2003; Lewis et al., 2004). This suggests that older subjects made more pervasive use of external information sources for controlling their limb movements by means of the metronome-pacing signal, 
Table 3. Brain regions additionally activated in the elderly as compared to the young subjects in which performance and brain activation is positively correlated in the OLD group during NONISODIR coordination

\begin{tabular}{llrrrr}
\hline Region activated & Side & $x$ & $y$ & $z$ & $t$ value \\
\hline Middle frontal gyrus (DLPFC) & $\mathrm{L}$ & -36 & 38 & 34 & 2.86 \\
Inferior frontal gyrus (pars opercularis/triangularis) & $\mathrm{L}$ & -48 & 20 & 24 & 4.67 \\
Anterior insula & $\mathrm{L}$ & -52 & 16 & -10 & 3.92 \\
Superior frontal sulcus (pre-PMd) & $\mathrm{L}$ & -36 & 4 & 54 & 3.71 \\
Superior temporal gyrus & $\mathrm{L}$ & -58 & -16 & -2 & 2.68 \\
Superior parietal gyrus & $\mathrm{L}$ & -18 & -62 & 56 & 3.42 \\
Cerebellar hemisphere (V) & $\mathrm{L}$ & -28 & -48 & -24 & 3.91 \\
Cerebellar hemisphere (VIIIB) & $\mathrm{R}$ & 18 & -60 & -50 & 3.40
\end{tabular}

The $t$ values and localizations (MNI coordinates) of activation peaks showing a significant ( $p<0.05$; cluster-wise FDR correction for multiple comparisons) positive correlation between the level of brain activity and coordination performance are shown. Regions reaching significance only at an uncorrected level $(p<0.001)$ are in italics. L, Left hemisphere; $R$, right hemisphere; $M$, medial.

which resulted in more accurate coordination performance. Furthermore, the IFGPO and IFGPT are involved in interfacing external information about biological motion with internal representations of limb actions, as observed during movement observation, imitation, or imagery (Grezes et al., 1998; Iacoboni et al., 1999; Binkofski et al., 2000; Molnar-Szakacs et al., 2005). The elevated activations in IFGPO and IFGPT therefore tentatively suggest that the successful elderly used some form of visualization strategy to control their movements. Together, the higher activations of these regions appear to reflect higher-level processing and integration of external and internal information sources to successfully guide motor coordination.

Finally, positive correlations between level of coordination performance and activation were observed in two regions in the contralateral frontal lobe (i.e., the pre-PMd and DLPFC). There is increasing agreement that the pre-PMd is more closely related to cognitive than to motor processes. More specifically, the prePMd is highly interconnected with the PFC (Lu et al., 1994; Geyer et al., 2000) and becomes activated with increasing cognitive demands of a motor task (for review, see Picard and Strick, 2001). The DLPFC receives visual, somatosensory, and auditory information from the occipital, temporal, and parietal cortices (Goldman-Rakic and Schwartz, 1982; Barbas and Pandya, 1989; Seltzer and Pandya, 1989; Pandya and Yeterian, 1990; Petrides and Pandya, 1999) and has preferential connections with the motor system structures (Miller and Cohen, 2001). Accordingly, the DLPFC is hypothesized to play a central role in the cognitive control of motor behavior (Miller and Cohen, 2001). Overall, the observed positive correlations between coordination accuracy and activation in the pre-PMd and DLPFC suggest that the older subjects relied on increased cognitive monitoring that had a beneficial impact on complex coordination performance, suggesting that the additional neural recruitment was compensatory.

The present findings are in partial agreement with several "cognitive aging" studies in which activation levels in frontal regions were shown to correlate positively with overall performance in the elderly (Reuter-Lorenz et al., 2000; Grady et al., 2003; Scarmeas et al., 2003; Madden et al., 2004; Rosano et al., 2005). Similarly, when groups of good versus bad performing elderly were compared, good performers exhibited more brain activation than bad performers (Cabeza et al., 2002; Rosen et al., 2002). In the present motor coordination study, the compensatory recruitment extended far beyond the prefrontal regions and involved a more extensive network, suggesting that such tasks are very instrumental to studying age-related alterations in complex brain function.

\section{Summary and conclusions}

Coordination in the elderly was associated with profound activations in (1) classical motor control regions, (2) higher-level sensorimotor regions reflecting increased reliance on sensory information processing, and (3) frontal regions reflecting increased cognitive control and performance monitoring. Importantly, the majority of these regions exhibited a positive correlation between brain activation level and performance in the elderly, such that increased recruitment in these regions was associated with higher motor coordination accuracy. This enabled some elderly to reach motor performance levels comparable to those obtained in the younger controls, suggesting that the additional recruitment is meaningful for preserving motor performance in the elderly (i.e., it is primarily compensatory in nature). However, compensatory recruitment might have a hidden cost. To the extent that older brains engage more neural circuitry at the same or lower levels of task demand than do younger adults, seniors may rely more on "cognitive reserve" (Scarmeas et al., 2003 ) and are thus more likely to reach a limit on the available resources that can be brought to bear on task performance (DiGirolamo et al., 2001; Reuter-Lorenz and Lustig, 2005; Reuter-Lorenz and Mikels, 2006; P. A. Reuter-Lorenz and K. A. Cappell, unpublished observation). This may perhaps explain why poorly performing older adults showed BOLD responses similar to those of the young participants, whereas the successful elderly exhibited more elaborate activation than young adults in some brain regions.

In contrast to recent cognitive aging studies in which compensatory recruitment was established in the prefrontal regions, the present study showed activation across a more elaborate network that was dedicated to increasing cognitive control and enhanced processing of sensory information for motor performance, indicative of systems-level neuroplasticity at higher age. This penetration of cognition into motor performance appears to be a marker of successful aging, and it paves the way for rehabilitation interventions that promote the exploitation of cognitive processing mechanisms for action control.

\section{References}

Baldissera F, Cavallari P, Civaschi P (1982) Preferential coupling between voluntary movements of ipsilateral limbs. Neurosci Lett 34:95-100.

Baldissera F, Cavallari P, Marini G, Tassone G (1991) Differential control of in-phase and anti-phase coupling of rhythmic movements of ipsilateral hand and foot. Exp Brain Res 83:375-380.

Bamiou DE, Musiek FE, Luxon LM (2003) The insula (Island of Reil) and its role in auditory processing. Literature review. Brain Res Brain Res Rev 42:143-154.

Barbas H, Pandya DN (1989) Architecture and intrinsic connections of the prefrontal cortex in the rhesus monkey. J Comp Neurol 286:353-375.

Binkofski F, Amunts K, Stephan KM, Posse S, Schormann T, Freund HJ, Zilles K, Seitz RJ (2000) Broca's region subserves imagery of motion: a combined cytoarchitectonic and fMRI study. Hum Brain Mapp 11:273-285.

Bushara KO, Wheat JM, Khan A, Mock BJ, Turski PA, Sorenson J, Brooks BR (2001) Multiple tactile maps in the human cerebellum. NeuroReport 12:2483-2486.

Cabeza R (2002) Hemispheric asymmetry reduction in older adults: the HAROLD model. Psychol Aging 17:85-100. 
Cabeza R, Anderson ND, Locantore JK, McIntosh AR (2002) Aging gracefully: compensatory brain activity in high-performing older adults. NeuroImage 17:1394-1402.

Calautti C, Serrati C, Baron JC (2001) Effects of age on brain activation during auditory-cued thumb-to-index opposition: a positron emission tomography study. Stroke 32:139-146.

Carson RG, Goodman D, Kelso JA, Elliott D (1995) Phase transitions and critical fluctuations in rhythmic coordination of ipsilateral hand and foot. J Mot Behav 27:211-224.

Debaere F, Swinnen SP, Beatse E, Sunaert S, Van Hecke P, Duysens J (2001) Brain areas involved in interlimb coordination: a distributed network. NeuroImage 14:947-958.

Debaere F, Wenderoth N, Sunaert S, Van Hecke P, Swinnen SP (2004) Cerebellar and premotor function in bimanual coordination: parametric neural responses to spatiotemporal complexity and cycling frequency. NeuroImage 21:1416-1427.

DiGirolamo GJ, Kramer AF, Barad V, Cepeda NJ, Weissman DH, Milham MP, Wszalek TM, Cohen NJ, Banich MT, Webb A, Belopolsky AV, McAuley E (2001) General and task-specific frontal lobe recruitment in older adults during executive processes: a fMRI investigation of taskswitching. NeuroReport 12:2065-2071.

Ehrsson HH, Naito E, Geyer S, Amunts K, Zilles K, Forssberg H, Roland PE (2000) Simultaneous movements of upper and lower limbs are coordinated by motor representations that are shared by both limbs: a PET study. Eur J Neurosci 12:3385-3398.

Folstein MF, Folstein SE, McHugh PR (1975) “Mini-mental state.” A practical method for grading the cognitive state of patients for the clinician. J Psychiatr Res 12:189-198.

Friston KJ, Holmes AP, Poline JB, Grasby PJ, Williams SC, Frackowiak RS, Turner R (1995a) Analysis of fMRI time-series revisited. NeuroImage 2:45-53.

Friston KJ, Holmes A, Worsley K, Poline J, Frith C, Frackowiak R (1995b) Statistical parametric maps in functional imaging: a general linear approach. Hum Brain Mapp 2:189-210.

Geyer S, Matelli M, Luppino G, Zilles K (2000) Functional neuroanatomy of the primate isocortical motor system. Anat Embryol (Berl) 202:443-474.

Goldman-Rakic PS, Schwartz ML (1982) Interdigitation of contralateral and ipsilateral columnar projections to frontal association cortex in primates. Science 216:755-757.

Grady CL (2002) Introduction to the special section on aging, cognition, and neuroimaging. Psychol Aging 17:3-6.

Grady CL, McIntosh AR, Beig S, Keightley ML, Burian H, Black SE (2003) Evidence from functional neuroimaging of a compensatory prefrontal network in Alzheimer's disease. J Neurosci 23:986-993.

Grezes J, Costes N, Decety J (1998) Top-down effect of strategy on the perception of human biological motion: a PET investigation. Cogn Neuropsychol 15:553-582.

Habas C, Axelrad H, Nguyen TH, Cabanis EA (2004) Specific neocerebellar activation during out-of-phase bimanual movements. NeuroReport 15:595-599.

Heuninckx S, Debaere F, Wenderoth N, Verschueren S, Swinnen SP (2004) Ipsilateral coordination deficits and central processing requirements associated with coordination as a function of aging. J Gerontol B Psychol Sci Soc Sci 59:225-232.

Heuninckx S, Wenderoth N, Debaere F, Peeters R, Swinnen SP (2005) Neural basis of aging: the penetration of cognition into action control. J Neurosci 25:6787-6796.

Iacoboni M, Woods RP, Brass M, Bekkering H, Mazziotta JC, Rizzolatti G (1999) Cortical mechanisms of human imitation. Science 286:2526-2528.

Ivry R (1997) Cerebellar timing systems. Int Rev Neurobiol 41:555-573.

Jueptner M, Ottinger S, Fellows SJ, Adamschewski J, Flerich L, Muller SP, Diener HC, Thilmann AF, Weiller C (1997) The relevance of sensory input for the cerebellar control of movements. NeuroImage 5:41-48.

Kelso JA, Jeka JJ (1992) Symmetry breaking dynamics of human multilimb coordination. J Exp Psychol Hum Percept Perform 18:645-668.

Lewis PA, Wing AM, Pope PA, Praamstra P, Miall RC (2004) Brain activity correlates differentially with increasing temporal complexity of rhythms during initialisation, synchronisation, and continuation phases of paced finger tapping. Neuropsychologia 42:1301-1312.

Li SC, Lindenberger U (1999) Cross-level unification: a computational exploration of the link between deterioration of neurotransmitter systems dedifferentiation of cognitive abilities in old age. In: Cognitive neuroscience of memory (Nilsson LG, Markowitsch HJ, eds), pp 103-146. Seattle: Hogrefe and Huber.

Lu MT, Preston JB, Strick PL (1994) Interconnections between the prefrontal cortex and the premotor areas in the frontal lobe. J Comp Neurol 341:375-392.

Macar F, Vidal F, Casini L (1999) The supplementary motor area in motor and sensory timing: evidence from slow brain potential changes. Exp Brain Res 125:271-280.

Madden DJ, Turkington TG, Provenzale JM, Denny LL, Hawk TC, Gottlob LR, Coleman RE (1999) Adult age differences in the functional neuroanatomy of verbal recognition memory. Hum Brain Mapp 7:115-135.

Madden DJ, Whiting WL, Provenzale JM, Huettel SA (2004) Age-related changes in neural activity during visual target detection measured by fMRI. Cereb Cortex 14:143-155.

Mattay VS, Fera F, Tessitore A, Hariri AR, Das S, Callicott JH, Weinberger DR (2002) Neurophysiological correlates of age-related changes in human motor function. Neurology 58:630-635.

Miller EK, Cohen JD (2001) An integrative theory of prefrontal cortex function. Annu Rev Neurosci 24:167-202.

Mima T, Sadato N, Yazawa S, Hanakawa T, Fukuyama H, Yonekura Y, Shibasaki H (1999) Brain structures related to active and passive finger movements in man. Brain 122:1989-1997.

Molnar-Szakacs I, Iacoboni M, Koski L, Mazziotta JC (2005) Functional segregation within pars opercularis of the inferior frontal gyrus: evidence from fMRI studies of imitation and action observation. Cereb Cortex 15:986-994.

Nichols T, Brett M, Andersson J, Wager T, Poline JB (2005) Valid conjunction inference with the minimum statisic. NeuroImage 25:653-660.

Oldfield RC (1971) The assessment and analysis of handedness: the Edinburgh inventory. Neuropsychologia 9:97-113.

Pandya DN, Yeterian EH (1990) Prefrontal cortex in relation to other cortical areas in rhesus monkey: architecture and connections. Prog Brain Res 85:63-94.

Petrides M, Pandya DN (1999) Dorsolateral prefrontal cortex: comparative cytoarchitectonic analysis in the human and the macaque brain and corticocortical connection patterns. Eur J Neurosci 11:1011-1036.

Picard N, Strick PL (2001) Imaging the premotor areas. Curr Opin Neurobiol 11:663-672.

Platel H, Price C, Baron JC, Wise R, Lambert J, Frackowiak RS, Lechevalier B, Eustache F (1997) The structural components of music perception. A functional anatomical study. Brain 120:229-243.

Reuter-Lorenz PA, Lustig C (2005) Brain aging: reorganizing discoveries about the aging mind. Curr Opin Neurobiol 15:245-251.

Reuter-Lorenz PA, Mikels J (2006) The aging brain: implications of enduring plasticity for behavioral and cultural change. In: Lifespan development and the brain: the perspective of biocultural co-constructivism (Baltes P, Reuter-Lorenz P, Roesler F, eds), p 255. Cambridge, UK: Cambridge UP.

Reuter-Lorenz PA, Jonides J, Smith EE, Hartley A, Miller A, Marshuetz C, Koeppe RA (2000) Age differences in the frontal lateralization of verbal and spatial working memory revealed by PET. J Cogn Neurosci 12:174-187.

Rosano C, Aizenstein H, Cochran J, Saxton J, De Kosky S, Newman AB, Kuller LH, Lopez OL, Carter CS (2005) Functional neuroimaging indicators of successful executive control in the oldest old. NeuroImage 28:881-889.

Rosen AC, Prull MW, O'Hara R, Race EA, Desmond JE, Glover GH, Yesavage JA, Gabrieli JD (2002) Variable effects of aging on frontal lobe contributions to memory. NeuroReport 13:2425-2428.

Scarmeas N, Zarahn E, Anderson KE, Hilton J, Flynn J, Van Heertum RL, Sackeim HA, Stern Y (2003) Cognitive reserve modulates functional brain responses during memory tasks: a PET study in healthy young and elderly subjects. NeuroImage 19:1215-1227.

Seltzer B, Pandya DN (1989) Intrinsic connections and architectonics of the superior temporal sulcus in the rhesus monkey. J Comp Neurol 290:451-471.

Serrien DJ, Swinnen SP, Stelmach GE (2000) Age-related deterioration of coordinated interlimb behavior. J Gerontol B Psychol Sci Soc Sci 55B:295-303.

Swinnen SP, Dounskaia N, Verschueren S, Serrien DJ, Daelman A (1995) 
Relative phase destabilization during interlimb coordination: the disruptive role of kinesthetic afferences induced by passive movement. Exp Brain Res 105:439-454.

Talairach J, Tournoux P (1988) Co-planar stereotaxic atlas of the human brain. New York: Thieme.

Thaut MH (2003) Neural basis of rhythmic timing networks in the human brain. Ann NY Acad Sci 999:364-373.

Thickbroom GW, Byrnes ML, Mastaglia FL (2003) Dual representation of the hand in the cerebellum: activation with voluntary and passive finger movement. NeuroImage 18:670-674.

Ward NS, Frackowiak RS (2003) Age-related changes in the neural correlates of motor performance. Brain 126:873-888.

Wenderoth N, Debaere F, Sunaert S, Van Hecke P, Swinnen SP (2004a)
Parieto-premotor areas mediate directional interference during bimanual movements. Cereb Cortex 14:1153-1163.

Wenderoth N, Debaere F, Swinnen SP (2004b) Neural networks involved in cyclical interlimb coordination as revealed by medical imaging techniques. In: Neuro-behavioral determinants of interlimb coordination: a multidisciplinary approach (Swinnen SP, Duysens J, eds), pp 127-222. Boston: Kluwer Academic.

Wenderoth N, Debaere F, Sunaert S, Swinnen SP (2005) The role of anterior cingulate cortex and precuneus in the coordination of motor behaviour. Eur J Neurosci 22:235-246.

Wenderoth N, Toni I, Bedeleem S, Debaere F, Swinnen SP (2006) Information processing in human parieto-frontal circuits during goal-directed bimanual movements. NeuroImage 31:264-278. 